\title{
Economic characterization of predominant farming systems in West Bengal, India
}

\author{
Soumitra Chatterjee ${ }^{1}$, Manabendra Ray ${ }^{2}$, Pallabendu Halder ${ }^{3}$, Rupak Goswami ${ }^{4}$ \\ ${ }^{1}$ Assistant Professor in Agricultural Economics, AICRP on IFS, BCKV, Kalyani, India \\ ${ }^{2}$ Assosciate Professor in Agronomy, AICRP on IFS, BCKV, Kalyani, India \\ ${ }^{3}$ Senior Research Fellow in Agronomy, AICRP on IFS, BCKV, Kalyani, India \\ ${ }^{4}$ Assistant Professor, IRDM Faculty Centre, Ramakrishna Mission Vivekananda University, Narendrapur, Kolkata, India
}

\section{Email address:}

soumitra.soum123123@gmail.com (S. Chatterjee)

\section{To cite this article:}

Soumitra Chatterjee, Manabendra Ray, Pallabendu Halder, Rupak Goswami. Economic Characterization of Predominant Farming Systems in West Bengal, India. American Journal of Agriculture and Forestry. Vol. 1, No. 3, 2013, pp. 40-47.

doi: 10.11648/j.ajaf.20130103.11

\begin{abstract}
This paper seeks to characterize and evaluate predominant farming systems in economic terms across all agroclimatic zones of West Bengal, India as well as to assess the extent of crop diversification among different size classes. A multi-stage random sampling method followed by a farm survey with structured interview schedule revealed that farming community of all size classes intended to shift from their traditional rice based cropping system to more income generating systems like fruits, vegetables, livestock, plantation, spices and piggery based sub-systems in Hilly tract, fibre and vegetables based systems in Terai and Old Alluvium regions, vegetables based systems in New Alluvial, sugarcane and vegetable based systems in Lateritic Red Soil zone and fishery, oilseeds and plantation (betel vine) based systems in the Coastal belt of the state. The extent of crop diversification was maximum among small and marginal farmers and a strong and continued shift towards vegetables and orchard crop was observed. The farmers across all regions consciously invested water and inputs in fruits and vegetables to achieve greater return in comparison to rice and other agronomic crops. Jute crop, despite of its oddities with price, was more of agronomic compulsion, which was visible in Terai and Old Alluvium tract. Jute rendered more benefits with vegetables than rice in the same piece of land. Despite of several odds in the problematic Lateritic Red Soil region of the state, sugarcane was identified as predominant crop with good economic return. Livestock rearing was also on the rise along with fishery in the problematic Coastal Saline belt. This has been a welcome shift and diversification of farm enterprise. With gradual departure from rice and other cereals and shift favouring vegetables, orchard, livestock and fishery enterprises farm families have better productivity and remuneration per unit of land and might provide employment opportunity with less gestation.
\end{abstract}

Keywords: Predominant Farming System, Economic Characterization, Crop Diversification, Economic Return

\section{Introduction}

Identification and characterization of farm types is of critical importance for precise technological intervention and policy support. Farming system is described as a unit consisting of a human group (usually a household) and the resources it manages in its environment, involving the direct production of plant and/or animal products [1]. Typology of farming system is dictated by climate, production goals and culture of a society. This classification of the farming situations of developing regions may be as varied as - available natural resource base, climate, landscape, farm size, tenure and organization, dominant pattern of farm activities and household livelihoods, which determine the intensity of production and integration of crops, livestock and other activities [2]. Unfortunately, both in agricultural and social sciences, complexity and diversity has remained undervalued, and excluded from government statistics and policy framework [3]. The heterogeneity across different regions as well as variation in different farms are influenced by a host of biophysical (e.g. climate, soil fertility, slope etc.) and/or socio-economic (e.g. preferences, prices, production objectives etc.) factors [4]. Developing a typology constitutes an essential step in any realistic evaluation of the constraints and opportunities that exists within farm households for forwarding appropriate 
policy interventions $[5,6,7]$. The factors that define farm typology vary greatly from study to study and/or from region to region. These may be as diverse as agroecological, socio-economic, managerial, infrastructural and broader issues of livelihoods [8].

With lapse of time, agriculture in many small farms, have assumed commercial proportion, replacing its traditionally subsistence nature. Hence, farm typology delineation based on monetary income seems to be a pragmatic approach. This will also save resources by significantly curtaining the volume of survey data. With economic characterization of farms as the objective at hand, the authors assume that classification of farms based on economic returns will give more effective insights regarding farm types.

Keeping this view in mind, an attempt was made to identify, characterize and evaluate predominant farming systems of West Bengal state, India to find out the economically profitable farming systems, its contributions to overall livelihood of the farming community and extent of crop diversification across all agro-climatic zones of the state

\section{Materials and Methods}

As per the detailed guidelines of Indian Agricultural Statistical Research Institute, Pusa, New Delhi, a multistage random sample survey method has been used to perform the entire survey work regarding characterization and evaluation of predominant farming systems across all National Agricultural Research Project (NARP) zones of West Bengal, India. According to NARP report, West Bengal has six agro-climatic zones namely: Hill Zone, Terai Zone, Old Alluvial Zone, New Alluvial Zone, Lateritic Red Soil Zone and Coastal Saline Zone (Fig. 1). From each zone one district each - one advanced and one backward in terms of existing cropping intensity - was taken for the study. From each district, four representative Blocks and three villages from each of the Block was selected randomly. Six representative farmers comprised of marginal-small, medium and large, in terms of operational holding size, were taken up randomly for the survey. The sampling scheme slightly swerved from the standard in pockets where marginal-small farming communities were predominant in nature. Further, the conventional size classification of the farming community prescribed by the department of Land Reform, Government of West Bengal was not followed precisely because of the overwhelming proportion of marginal-small farming community in backward zones of the State. Overall, marginal farming community ( $<0.67$ ha.), small (0.67-1.0 ha.), medium (1.01.33 ha.) and large one ( $>1.33$ ha.) have been framed purposively for the convenience of the survey work. Seventy two farming communities from each district and 144 from each NARP zone (except Hill zone) was surveyed, classified and categorized for the identification of the predominant farming system. Thus, a total of 792 farms were surveyed.

The predominant farming system was identified based on the highest gross income received from a farming system component. In identifying sub-system, component contributing highest income was followed by the component contributing next best income to the system, and so on. The name of components in a given sub-system has been put as per their contributions to the whole farm gross income. For example, sub-system 'Rice + Jute + Vegetables' means that Rice contributes highest to the farm income followed by Jute and Vegetables. Economics of different farming systems has also been calculated to evaluate economically most profitable system for each zone.

The following figure represents the redefined six agroclimatic zones of West Bengal, India where zone 1 to 6 has been demarcated with different colours where, zone 1 denotes Northern Hill Zone (29.1K sq. Km) comprises of entire Darjeeling district excluding Siliguri sub-division and small part of Jalpaiguri. Zone 2 represents Terai Teesta Flood plain of Bengal ( $12 \mathrm{~K}$ sq. $\mathrm{Km})$ comprises of Coochbehar, Jalpaiguri and part of Uttar Dinajpur districts. Zone 3 represents the Gangetic Flood plain (16.3K sq. Km) including part of Dakshin Dinajpur, Malda, Uttar Dinajpur, Burdwan, Murshidabad, 24-Parganas (North), 24-Parganas (South), Howrah, Hooghly, Birbhum and the entire Nadia district. Zone 4 denotes the Undulating Lateritic region (23.8K sq. Km) comprises of (entire Purulia district and part of Burdwan, Birbhum, Bankura and West Midnapore). Zone 5 represents Vindhyan Old Flood plain (17.9K sq. Km) comprises of part of Murshidabad, Howrah, Hooghly, Burdwan, Birbhum, West and East Midnapore). Finally zone 6 denotes the Coastal Saline zone (12.8K sq. Km) comprises of major part of 24-Parganas (South) and East Midnapore and a small part of Howrah and 24-Parganas (North).

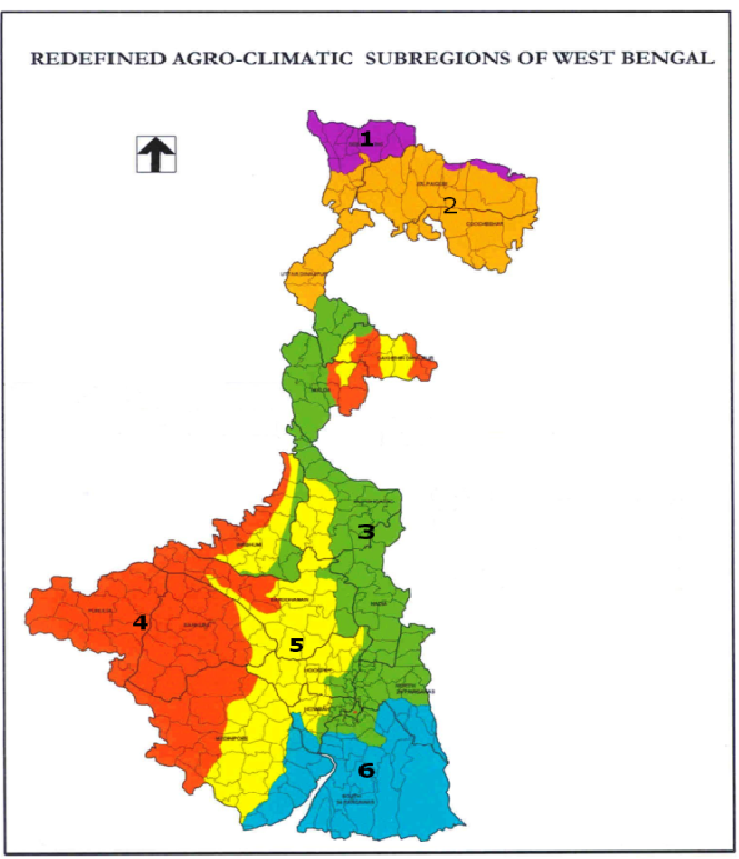




\subsection{Data}

Based on an extensive review of literature, initial case explorations in the field and expert counsel, a structured interview schedule was developed for the study. Apart from socio-economic parameters of the households, farm size, existing and emerging farming systems of the sample households, information on cost of cultivation and yield of different crops and price received by the farmers was included in the data collection instrument. The draft interview schedule was then pre-tested on 15 non-sampled respondents of each agro-climatic zone to incorporate necessary modifications in the instrument. Two of the important variables and their measurement is described below -

\subsection{Cost Benefit Analysis}

For measuring the production efficiency benefit cost ratio can be worked out as follows

Benefit cost ratio $=\frac{\text { Gross return from sale of output }}{\text { Total cost of input used }}$

\subsection{Measurement of Crop Diversification}

Herfindahal index as diversification measures have been used in the following form:

$$
\mathrm{HI}=\sum_{\mathrm{i}=1}^{\mathrm{n}} \mathrm{Pi}^{2}
$$

Where, $\mathrm{Pi}=\mathrm{Ai} / \sum_{i=1}^{n} A i, \mathrm{i}=1,2,3 \ldots \ldots$. Number of crop enterprises. $\mathrm{Pi}=$ proportion of area under crop, $\mathrm{Ai}=$ area under $i^{\text {th }}$ crop and $\sum A \mathrm{i}=$ gross cropped area of the farm. The value of Herfindahal index varies from 0 to 1 where 1 indicates complete specialization and 0 indicates perfect diversification.

\section{Results and Discussion}

\subsection{Farm Size-Wise Identification of Predominant Farming Systems}

Farm-size wise predominant farming systems across all agro-climatic zones of West Bengal was identified that showed that Hill zone was dominated by vegetable based farming system (38.89\%) followed by dairy (20.83\%) and plantation based systems (9.72\%). Marginal-small farmers, dominant in this zone, adopted diversified farming system apart from growing traditional cereal crops. Terai zone was dominated by conventional cereal-based farming systems (41\%), followed by vegetable based sub-system (35\%), and jute based systems (8.33\%). In Old Alluvial zone, a zone situated in the Gangetic alluvium tract and one of the high productivity zones of the state, $36.81 \%$ households followed cereal based farming systems. Dairy was found to be the next popular system adopted by the farming community. New alluvial zone was predominated by vegetable-based farming systems $(90.28 \%)$. In many parts of this region, departure from traditional cropping pattern has been a trend for the last one and half decades $[9,10]$ mostly for enhancing income from fragmented land resources. Vegetable based farming system was also predominant in the Red Lateritic region (41.67\% contribution to total) of the state, which was known to be one of the problematic zones of the state with unfavourable water regime, topography, soil fertility and socio-economic condition of the farm families. The region is known to be the dry tract of Bengal where rainfed rice was cultivated as monocrop. Barring scarcity in irrigation water, sugarcane was identified as a predominant crop in this region with $13.89 \%$ farm households following this system. Another problematic tract of the state is Coastal belt of Bengal where high soil salinity results into poor agricultural production. Rainfed rice has been registered as the most predominant farming system in this region that covers around $48.0 \%$ of the farm family, followed by fishery based sub systems $(26.39 \%$ contribution to total). The region is well connected to Kolkata and non-farm activities have a significant effect on socio-economic status and rural livelihoods of the farm families of the zone $(11.11 \%$ of total farming community surveyed) (Table 1). This is not surprising since non-farm income has become a burgeoning reality of rural India especially for the smallholders and this has often become the largest source of rural farm income [10].

Table 1: Farm size-wise number of farmers having different farming systems in all NARP Zones of West Bengal

\begin{tabular}{|c|c|c|c|c|c|c|c|c|c|c|c|c|c|c|c|c|c|c|c|c|c|c|c|c|}
\hline \multirow{2}{*}{$\begin{array}{l}\text { Catego } \\
\text { ry }\end{array}$} & \multicolumn{4}{|c|}{ Hill Zone } & \multicolumn{4}{|c|}{ Terai Zone } & \multicolumn{4}{|c|}{ Old Alluvial Zone } & \multicolumn{4}{|c|}{ New Alluvial Zone } & \multicolumn{4}{|c|}{ Lateritic \& Red Soil Zone } & \multicolumn{4}{|c|}{ Coastal Saline Zone } \\
\hline & $\begin{array}{l}\text { Mar- } \\
\text { Small }\end{array}$ & $\begin{array}{c}\text { Medi } \\
\text { um }\end{array}$ & Large & All & $\begin{array}{l}\text { Mar- } \\
\text { Small }\end{array}$ & $\begin{array}{c}\text { Medi } \\
\text { um }\end{array}$ & Large & All & $\begin{array}{c}\text { Mar- } \\
\text { Small }\end{array}$ & $\begin{array}{c}\text { Medi } \\
\text { um }\end{array}$ & Large & All & $\begin{array}{c}\text { Margi } \\
\text { nal }\end{array}$ & Small & $\begin{array}{c}\mathrm{Med} / \mathrm{La} \\
\text { rge }\end{array}$ & All & $\underset{\text { nal }}{\text { Margi }}$ & i Small & $\begin{array}{c}\mathrm{Med} / \mathrm{La} \\
\mathrm{rge}\end{array}$ & All & $\underset{\text { nal }}{\text { Margi }}$ & Small & $\begin{array}{c}\text { Med/La } \\
\text { rge }\end{array}$ & All \\
\hline & 3 & 2 & 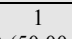 & 6 & 18 & 20 & 21 & . & 17 & 17 & (1) & - & & & & & & 17 & & & & (1) & & 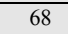 \\
\hline & $(6.00)$ & 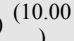 & 0.0 & $(8.33)$ & $(36.73$ & 55 & 75 & $(40.97$ & 7( & $(36.96$ & 6( & $(3$ & $(6.52)$ & (1.59) & $(0.00)$ & $(2.78)$ & $(47.27)$ & $(45.16$ & $(15.52)$ & 03 & $(40.0$ & $(46.34$ & $(63.64)$ & $(47.22$ \\
\hline 'egeta & 21 & 7 & & 28 & ) & 18 & 18 & 49 & ) & ) & ) & ) & & & & & & ) & & & & & & \\
\hline & $(42.00$ & $(35.00$ & 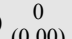 & $(38.89$ & $(26.53$ & $(38.30$ & $(37.50$ & $(34.03$ & 2 & 1 & 1 & 4 & 41 & $\begin{array}{c}58 \\
(92.06\end{array}$ & 31 & $(90.28$ & 22 & 29.03 & 29 & $\begin{array}{c}00 \\
(41.67\end{array}$ & 5 & 1 & 1 & 7 \\
\hline ed & ) & ) & $(0.00$ & ) & ) & ) & ) & ) & (3. & 7) & $(2.33)$ & $(2.78)$ & $(89.13)$ & ) & (88.5 & & & 2,0 & $(50.0$ & & 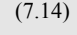 & 4) & 3) & 6) \\
\hline seed & 0 & 0 & 0 & 0 & 0 & 0 & 0 & 0 & 0 & 1 & 1 & 2 & 0 & 0 & 0 & 0 & 4 & 1 & . & 6 & 1 & 0 & 1 & 2 \\
\hline $\begin{array}{l}\text { lses } \\
\text { sed }\end{array}$ & $\begin{array}{c}0 \\
(0.00)\end{array}$ & $\begin{array}{c}0 \\
(0.00)\end{array}$ & $\begin{array}{c}0 \\
(0.00)\end{array}$ & $\begin{array}{c}0 \\
(0.00)\end{array}$ & $\begin{array}{c}0 \\
(0.00)\end{array}$ & $\begin{array}{c}0 \\
(0.00)\end{array}$ & $\begin{array}{c}0 \\
(0.00)\end{array}$ & $\begin{array}{c}0 \\
(0.00)\end{array}$ & $\begin{array}{c}3 \\
(5.45)\end{array}$ & $\begin{array}{c}2 \\
(4.35)\end{array}$ & $\begin{array}{c}1 \\
(2.33)\end{array}$ & $\begin{array}{c}6 \\
(4.17)\end{array}$ & $\begin{array}{c}0 \\
(0.00)\end{array}$ & $\begin{array}{c}0 \\
(0.00)\end{array}$ & $\begin{array}{c}0 \\
(0.00)\end{array}$ & $\begin{array}{c}0 \\
(0.00)\end{array}$ & $\begin{array}{c}0 \\
(0.00)\end{array}$ & $\begin{array}{c}0 \\
(0.00)\end{array}$ & $\begin{array}{c}1 \\
(1.72)\end{array}$ & $\begin{array}{c}1 \\
(0.69)\end{array}$ & $\begin{array}{c}0 \\
(0.00)\end{array}$ & $\begin{array}{c}2 \\
(4.88)\end{array}$ & $\begin{array}{c}0 \\
(0.00)\end{array}$ & $\begin{array}{c}2 \\
(1.39)\end{array}$ \\
\hline $\begin{array}{l}\text { Sugarc } \\
\text { ane } \\
\text { based }\end{array}$ & $\begin{array}{c}0 \\
(0.00)\end{array}$ & $\begin{array}{c}0 \\
(0.00)\end{array}$ & $\begin{array}{c}0 \\
(0.00)\end{array}$ & $\begin{array}{c}0 \\
(0.00)\end{array}$ & $\begin{array}{c}0 \\
(0.00)\end{array}$ & $\begin{array}{c}0 \\
(0.00)\end{array}$ & $\begin{array}{c}0 \\
(0.00)\end{array}$ & $\begin{array}{c}0 \\
(0.00)\end{array}$ & $\begin{array}{c}0 \\
(0.00)\end{array}$ & $\begin{array}{c}0 \\
(0.00)\end{array}$ & $\begin{array}{c}0 \\
(0.00)\end{array}$ & $\begin{array}{c}0 \\
(0.00)\end{array}$ & $\begin{array}{c}0 \\
(0.00)\end{array}$ & $\begin{array}{c}0 \\
(0.00)\end{array}$ & $\begin{array}{c}0 \\
(0.00)\end{array}$ & $\begin{array}{c}0 \\
(0.00)\end{array}$ & $\begin{array}{c}1 \\
(1.82)\end{array}$ & $\begin{array}{c}5 \\
(16.13 \\
)\end{array}$ & $\begin{array}{c}14 \\
(24.14)\end{array}$ & $\begin{array}{c}20 \\
(13.89 \\
)\end{array}$ & $\begin{array}{c}0 \\
(0.00)\end{array}$ & $\begin{array}{c}0 \\
(0.00)\end{array}$ & $\begin{array}{c}0 \\
(0.00)\end{array}$ & $\begin{array}{c}0 \\
(0.00)\end{array}$ \\
\hline & $\begin{array}{c}0 \\
(0.00)\end{array}$ & $\begin{array}{c}0 \\
(0.00)\end{array}$ & $\begin{array}{c}0 \\
(0.00)\end{array}$ & $\begin{array}{c}0 \\
(0.00)\end{array}$ & $\begin{array}{c}3 \\
(6.12)\end{array}$ & $\begin{array}{c}5 \\
(10.64 \\
)\end{array}$ & $\begin{array}{c}4 \\
(8.33)\end{array}$ & $\begin{array}{c}12 \\
(8.33)\end{array}$ & $\begin{array}{c}3 \\
(5.45)\end{array}$ & $\begin{array}{c}2 \\
(4.35)\end{array}$ & $\begin{array}{c}2 \\
(4.65)\end{array}$ & $\begin{array}{c}7 \\
(4.86)\end{array}$ & $\begin{array}{c}1 \\
(2.17)\end{array}$ & $\begin{array}{c}0 \\
(0.00)\end{array}$ & $\begin{array}{c}2 \\
(5.71)\end{array}$ & $\begin{array}{c}3 \\
(2.08)\end{array}$ & $\begin{array}{c}0 \\
(0.00)\end{array}$ & $\begin{array}{c}0 \\
(0.00)\end{array}$ & $\begin{array}{c}0 \\
(0.00)\end{array}$ & $\begin{array}{c}0 \\
(0.00)\end{array}$ & $\begin{array}{c}0 \\
(0.00)\end{array}$ & $\begin{array}{c}0 \\
(0.00)\end{array}$ & $\begin{array}{c}0 \\
(0.00)\end{array}$ & $\begin{array}{c}0 \\
(0.00)\end{array}$ \\
\hline & $\begin{array}{c}14 \\
(28.00\end{array}$ & $\begin{array}{c}1 \\
(5.00)\end{array}$ & $\begin{array}{c}0 \\
(0.00)\end{array}$ & $\begin{array}{c}15 \\
(20.83\end{array}$ & $\begin{array}{c}0 \\
(0.00)\end{array}$ & $\begin{array}{c}0 \\
(0.00)\end{array}$ & $\begin{array}{c}1 \\
(2.08)\end{array}$ & $\begin{array}{c}1 \\
(0.69)\end{array}$ & $\begin{array}{c}17 \\
(30.91\end{array}$ & $\begin{array}{c}17 \\
(36.96\end{array}$ & $\begin{array}{c}10 \\
6(23.26\end{array}$ & $\begin{array}{c}44 \\
(30.56\end{array}$ & $\begin{array}{c}0 \\
(0.00)\end{array}$ & $\begin{array}{c}2 \\
(3.17)\end{array}$ & $\begin{array}{c}2 \\
(5.71)\end{array}$ & $\begin{array}{c}4 \\
(2.78)\end{array}$ & $\begin{array}{c}1 \\
(1.82)\end{array}$ & $\begin{array}{c}1 \\
(3.23)\end{array}$ & $\begin{array}{c}0 \\
(0.00)\end{array}$ & $\begin{array}{c}2 \\
(1.39)\end{array}$ & $\begin{array}{c}1 \\
(1.43)\end{array}$ & $\begin{array}{c}0 \\
(0.00)\end{array}$ & $\begin{array}{c}0 \\
(0.00)\end{array}$ & $\begin{array}{c}1 \\
(0.69)\end{array}$ \\
\hline
\end{tabular}




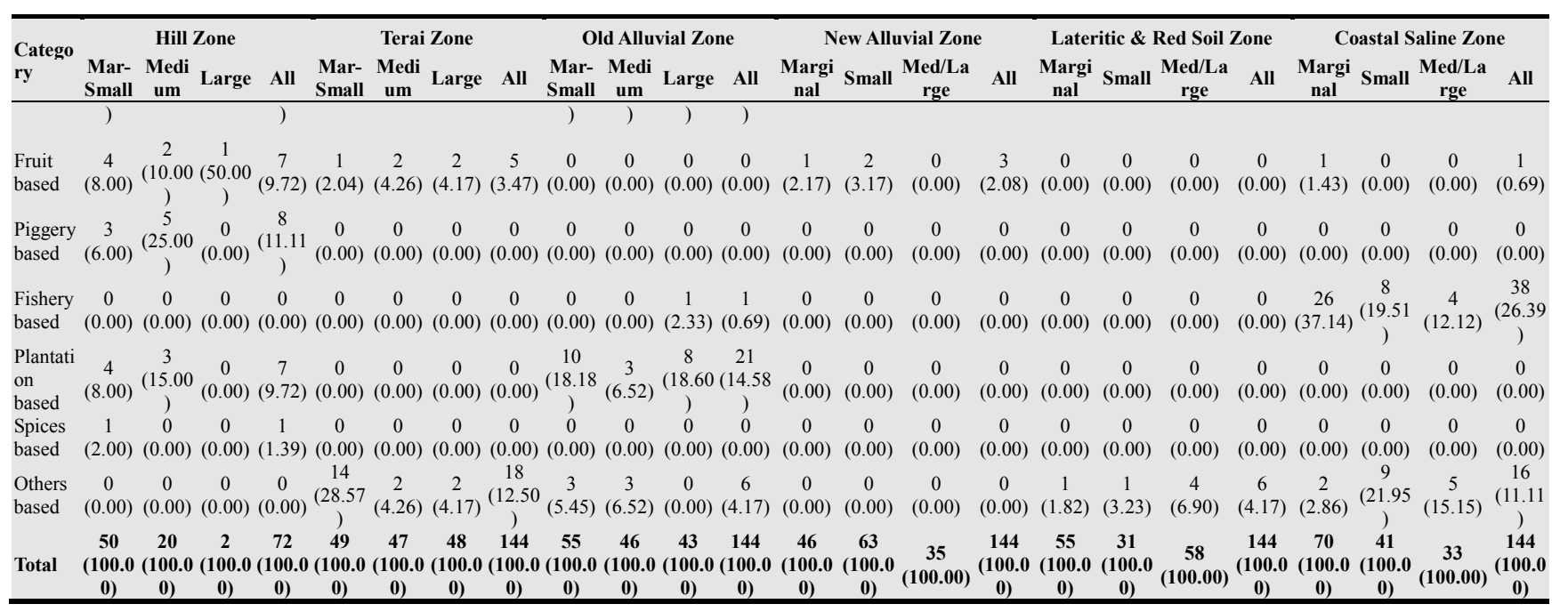

Figures in the parentheses indicates percentage to total

\subsection{Farm Size Wise Share of Gross Income Received from Different Farming Systems}

Cereal based sub-systems existed across all the regions of the state, highest share being in the Lateritic Red Soil zone with $83.92 \%$ contribution to gross farm income. The share in Coastal saline zone was $66.37 \%$. These two problematic regions suffered from climatic variability, topography and soil structure, and were known to be a monocrop region, rainfed rice being the sole crop cultivated. Next to cereals, vegetables based farming systems existed in all the zones. Hill zone demonstrated highest share $(89.06 \%)$ followed by Old alluvial $(83.09 \%)$, Coastal $(82.96 \%)$ and Red lateritic soil zone $(81.42 \%)$. Oilseeds based sub-systems showed $66.80 \%$ share in Coastal zone, where sunflower and sesame were predominant crops. Close to the Coastal region was Old alluvial tract (66.02\%). Pulse-based farming system existed in Old alluvial, Lateritic red soil and Coastal belt of Bengal, highest percent share being registered in Old alluvium tract (64.40\%) followed by Coastal (51.73\%) and Lateritic red soil zone $(48.35 \%)$. Sugarcane-based farming system shared $77.31 \%$ contribution to gross farm income in Lateritic red soil tract, while contribution of Jute-based farming system in Terai tract was $48.86 \%$. Dairy-based farming systems were observed to have developed in almost all the zones of the state. Hill and Old alluvial zones had maximum number of farming community under livestock and cattle rearing. Contribution to graoss farm income was highest in Hill $(56.65 \%)$ followed by Old alluvium tract $(41.16 \%)$. Fruit-based systems developed in
Hilly tract of Bengal (49.61\% share) where orange was found to be the most profitable fruit in terms of economic return. Piggery-based sub-system was observed in Hill zone with a share of $63.30 \%$. Fishery dominated in the Coastal belt of the state exhibiting a $68.18 \%$ share across all size classes of farm families. Plantation-based systems were predominant in Hilly tract of Bengal, tea plantation being the highest in making monetary return to the gross farm income. Overall, a 55.50\% share of gross income received from the plantation-based farming systems in this zone. Coastal belt of the state is famous for betel vine cultivation that contributed $83.41 \%$ of the gross income from plantation based farming systems. Spice based farming systems was found in Hilly regions (34.94\% share), cardamom, black pepper, cumin being the significant contributors. Barring Terai zone, contribution of non-farm income to the overall income was found in area adjacent to Kolkata city, where the largest number of labour forces came from the rural areas of New alluvial and Coastal zones. In Terai region, bamboo cultivators, wicker baskets producer and willow wood cutters were significant nonfarm occupations (Table 2). It was the marginal-small farming communities, who showed tendency to diversify their predominant farming systems into cash earning systems for improving their livelihood status. This might be due to the pressing need of increasing farm income from small fragmented holdings. The number of smallholders has increased in West Bengal [11] and there is also evidence of increased crop diversity on fragmented lands in the state [12].

Table 2: Farm size-wise percent share of gross income received from different systems in all NARP Zones of West Bengal

\begin{tabular}{|c|c|c|c|c|c|c|c|c|c|c|c|c|c|c|c|c|c|c|c|c|c|c|c|c|}
\hline \multirow[b]{2}{*}{ Category } & \multicolumn{2}{|c|}{$\begin{array}{l}\text { Hill Zone } \\
\text { Ma }\end{array}$} & \multirow[b]{2}{*}{$\begin{array}{l}\text { Lar } \\
\text { ge }\end{array}$} & \multirow[b]{2}{*}{ All } & \multicolumn{2}{|c|}{$\begin{array}{l}\text { Terai Zone } \\
\text { Mar }\end{array}$} & \multirow[b]{2}{*}{$\begin{array}{l}\text { Lar } \\
\text { ge }\end{array}$} & \multirow[b]{2}{*}{ All } & \multicolumn{3}{|c|}{$\begin{array}{l}\text { Old Alluvial Zone } \\
\text { Mar }\end{array}$} & \multirow[b]{2}{*}{ All } & \multicolumn{3}{|c|}{ New Alluvial Zone } & \multirow[b]{2}{*}{ All } & \multicolumn{4}{|c|}{ Lateritic \& Red Soil Zone } & \multicolumn{3}{|c|}{ Coastal Saline Zone } & \multirow[b]{2}{*}{ All } \\
\hline & $\begin{array}{l}\mathrm{r}- \\
\text { Sm } \\
\text { all }\end{array}$ & $\begin{array}{l}\text { Medi } \\
\text { um }\end{array}$ & & & $\begin{array}{l}- \\
\text { Sma } \\
\text { ll }\end{array}$ & $\begin{array}{l}\text { Medi } \\
\text { um }\end{array}$ & & & $\begin{array}{l}- \\
\text { Sma } \\
\text { II }\end{array}$ & $\begin{array}{l}\text { Medi } \\
\text { um }\end{array}$ & $\begin{array}{l}\text { Larg } \\
\text { e }\end{array}$ & & $\begin{array}{l}\text { Margi } \\
\text { nal }\end{array}$ & $\begin{array}{l}\text { Sma } \\
\text { Il }\end{array}$ & $\begin{array}{l}\text { Med/L } \\
\text { arge }\end{array}$ & & $\begin{array}{l}\text { Margi } \\
\text { nal }\end{array}$ & $\begin{array}{l}\text { Sm } \\
\text { all }\end{array}$ & $\begin{array}{l}\text { Med/L } \\
\text { arge }\end{array}$ & All & $\begin{array}{l}\text { Margi } \\
\text { nal }\end{array}$ & $\begin{array}{l}\text { Smal } \\
1\end{array}$ & & \\
\hline $\begin{array}{l}\text { Cereal } \\
\text { based }\end{array}$ & $\begin{array}{l}48 . \\
34 \\
(0.7 \\
0)\end{array}$ & $\begin{array}{l}58.65 \\
(1.40)\end{array}$ & $\begin{array}{l}38 . \\
44 \\
(3.2 \\
0)\end{array}$ & $\begin{array}{l}46 . \\
09 \\
(1.3 \\
5)\end{array}$ & $\begin{array}{l}47.3 \\
6 \\
(0.5 \\
3)\end{array}$ & $\begin{array}{l}49.26 \\
(1.05)\end{array}$ & $\begin{array}{l}59.6 \\
3 \\
(2.4 \\
5)\end{array}$ & $\begin{array}{l}55.5 \\
7 \\
(1.3 \\
9)\end{array}$ & $\begin{array}{l}69.8 \\
7 \\
(0.39 \\
6)\end{array}$ & $\begin{array}{l}44.62 \\
(1.98)\end{array}$ & $\begin{array}{l}50.8 \\
9 \\
(2.13 \\
0)\end{array}$ & $\begin{array}{l}51.8 \\
6 \\
(1.50 \\
2)\end{array}$ & $\begin{array}{l}53.91 \\
(0.352 \\
)\end{array}$ & $\begin{array}{l}46.5 \\
9 \\
(0.7 \\
92)\end{array}$ & $\begin{array}{l}0.00 \\
(0.00)\end{array}$ & $\begin{array}{l}52.8 \\
8 \\
(0.4 \\
62)\end{array}$ & $\begin{array}{l}85.48 \\
(0.35)\end{array}$ & $\begin{array}{l}79 . \\
02 \\
(0.7 \\
8)\end{array}$ & $\begin{array}{l}100.00 \\
(1.42)\end{array}$ & $\begin{array}{l}83 . \\
92 \\
(0.8 \\
5)\end{array}$ & $\begin{array}{l}76.50 \\
(0.28)\end{array}$ & $\begin{array}{l}60.92 \\
(0.74)\end{array}$ & $\begin{array}{l}60.54 \\
(1.16)\end{array}$ & $\begin{array}{l}66 . \\
37 \\
(0.5 \\
7)\end{array}$ \\
\hline Vegetable & 91. & 80.55 & 0.0 & 89. & 52.1 & 54.75 & 57.2 & 56.2 & 88.5 & 79.51 & 74.6 & 83.0 & 64.90 & 72.1 & 76.69 & 72.5 & 83.73 & 83. & 79.95 & 81. & 77.67 & 55.93 & 91.46 & 82. \\
\hline
\end{tabular}




\begin{tabular}{|c|c|c|c|c|c|c|c|c|c|c|c|c|c|c|c|c|c|c|c|c|c|c|c|c|}
\hline \multirow[b]{2}{*}{ Category } & \multicolumn{2}{|c|}{$\begin{array}{l}\text { Hill Zone } \\
\text { Ma }\end{array}$} & \multirow[b]{2}{*}{$\begin{array}{l}\text { Lar } \\
\text { ge }\end{array}$} & \multirow[b]{2}{*}{ All } & \multicolumn{2}{|c|}{$\begin{array}{l}\text { Terai Zone } \\
\text { Mar }\end{array}$} & \multirow[b]{2}{*}{$\begin{array}{l}\text { Lar } \\
\text { ge }\end{array}$} & \multirow[b]{2}{*}{ All } & \multicolumn{3}{|c|}{$\begin{array}{l}\text { Old Alluvial Zone } \\
\text { Mar }\end{array}$} & \multirow[b]{2}{*}{ All } & \multicolumn{3}{|c|}{ New Alluvial Zone } & \multirow[b]{2}{*}{ All } & \multicolumn{4}{|c|}{ Lateritic \& Red Soil Zone } & \multicolumn{4}{|c|}{ Coastal Saline Zone } \\
\hline & $\begin{array}{l}\mathbf{r}- \\
\text { Sm } \\
\text { all }\end{array}$ & $\begin{array}{l}\text { Medi } \\
\text { um }\end{array}$ & & & $\begin{array}{l}- \\
\text { Sma } \\
\text { ll }\end{array}$ & $\begin{array}{l}\text { Medi } \\
\text { um }\end{array}$ & & & $\begin{array}{l}- \\
\text { Sma } \\
\text { II }\end{array}$ & $\begin{array}{l}\text { Medi } \\
\text { um }\end{array}$ & $\begin{array}{l}\text { Larg } \\
\text { e }\end{array}$ & & $\begin{array}{l}\text { Margi } \\
\text { nal }\end{array}$ & $\begin{array}{l}\text { Sma } \\
\text { II }\end{array}$ & $\begin{array}{l}\mathrm{Med} / \mathrm{L} \\
\text { arge }\end{array}$ & & $\begin{array}{l}\text { Margi } \\
\text { nal }\end{array}$ & $\begin{array}{l}\text { Sm } \\
\text { all }\end{array}$ & $\begin{array}{l}\text { Med/L } \\
\text { arge }\end{array}$ & All & $\begin{array}{l}\text { Margi } \\
\text { nal }\end{array}$ & $\begin{array}{l}\text { Smal } \\
1\end{array}$ & $\begin{array}{l}\text { Med/L } \\
\text { arge }\end{array}$ & All \\
\hline based & $\begin{array}{l}99 \\
(0.5 \\
6)\end{array}$ & $(1.46)$ & $\begin{array}{l}0 \\
(0.0 \\
0)\end{array}$ & $\begin{array}{l}06 \\
(0.7 \\
9)\end{array}$ & $\begin{array}{l}8 \\
(0.4 \\
85)\end{array}$ & $(1.00)$ & $\begin{array}{l}3 \\
(2.0 \\
89)\end{array}$ & $\begin{array}{l}6 \\
(1.3 \\
02)\end{array}$ & $\begin{array}{l}7 \\
(0.79 \\
2)\end{array}$ & $\begin{array}{l}(1.58 \\
4)\end{array}$ & $\begin{array}{l}4 \\
(2.01 \\
8)\end{array}$ & $\begin{array}{l}9 \\
(1.46 \\
5)\end{array}$ & )$^{(0.375}$ & $\begin{array}{l}9 \\
(0.7 \\
91)\end{array}$ & $.367)$ & $\begin{array}{l}1 \\
(0.8 \\
14)\end{array}$ & .43) & $\begin{array}{l}19 \\
(0.8 \\
3)\end{array}$ & 7) & $\begin{array}{l}42 \\
(0.7 \\
8)\end{array}$ & 6) & $0.55)$ & $(1.66)$ & $\begin{array}{l}96 \\
(0.7 \\
2)\end{array}$ \\
\hline $\begin{array}{l}\text { Oilseeds } \\
\text { based }\end{array}$ & $\begin{array}{l}0.0 \\
0 \\
(0.0 \\
0)\end{array}$ & $\begin{array}{l}0.00 \\
(0.00)\end{array}$ & $\begin{array}{l}0.0 \\
0 \\
(0.0 \\
0)\end{array}$ & $\begin{array}{l}0.0 \\
0 \\
(0.0 \\
0)\end{array}$ & $\begin{array}{l}0.00 \\
(0.0 \\
0)\end{array}$ & $\begin{array}{l}0.00 \\
(0.00)\end{array}$ & $\begin{array}{l}0.00 \\
(0.0 \\
0)\end{array}$ & $\begin{array}{l}0.00 \\
(0.0 \\
0)\end{array}$ & $\begin{array}{l}0.00 \\
(0.00 \\
)\end{array}$ & $\begin{array}{l}53.92 \\
(1.42 \\
2)\end{array}$ & $\begin{array}{l}69.5 \\
7 \\
(2.31 \\
5)\end{array}$ & $\begin{array}{l}66.0 \\
2 \\
(1.24 \\
6)\end{array}$ & $\begin{array}{l}0.00 \\
(0.00)\end{array}$ & $\begin{array}{l}0.00 \\
(0.0 \\
0)\end{array}$ & $\begin{array}{l}0.00 \\
(0.00)\end{array}$ & $\begin{array}{l}0.00 \\
(0.0 \\
0)\end{array}$ & $\begin{array}{l}61.34 \\
(0.40)\end{array}$ & $\begin{array}{l}77 . \\
66 \\
(0.6 \\
4)\end{array}$ & $\begin{array}{l}51.80 \\
(1.40)\end{array}$ & $\begin{array}{l}58 . \\
44 \\
(0.8 \\
1)\end{array}$ & $\begin{array}{l}40.95 \\
(0.45)\end{array}$ & $\begin{array}{l}0.00 \\
(0.00)\end{array}$ & $\begin{array}{l}80.55 \\
(1.58)\end{array}$ & $\begin{array}{l}66 . \\
80 \\
(0.6 \\
1)\end{array}$ \\
\hline $\begin{array}{l}\text { Pulses } \\
\text { based }\end{array}$ & $\begin{array}{l}0.0 \\
0 \\
(0.0 \\
0)\end{array}$ & $\begin{array}{l}0.00 \\
(0.00)\end{array}$ & $\begin{array}{l}0.0 \\
0 \\
(0.0 \\
0)\end{array}$ & $\begin{array}{l}0.0 \\
0 \\
(0.0 \\
0)\end{array}$ & $\begin{array}{l}0.00 \\
(0.0 \\
0)\end{array}$ & $\begin{array}{l}0.00 \\
(0.00)\end{array}$ & $\begin{array}{l}0.00 \\
(0.0 \\
0)\end{array}$ & $\begin{array}{l}0.00 \\
(0.0 \\
0)\end{array}$ & $\begin{array}{l}65.7 \\
8 \\
(0.59 \\
4)\end{array}$ & $\begin{array}{l}66.68 \\
(1.41 \\
4)\end{array}$ & $\begin{array}{l}57.0 \\
7 \\
(2.13 \\
3)\end{array}$ & $\begin{array}{l}64.4 \\
0 \\
(1.38 \\
0)\end{array}$ & $\begin{array}{l}0.00 \\
(0.00)\end{array}$ & $\begin{array}{l}0.00 \\
(0.0 \\
0)\end{array}$ & $\begin{array}{l}0.00 \\
(0.00)\end{array}$ & $\begin{array}{l}0.00 \\
(0.0 \\
0)\end{array}$ & $\begin{array}{l}0.00 \\
(0.00)\end{array}$ & $\begin{array}{l}0.0 \\
0 \\
(0.0 \\
0)\end{array}$ & $\begin{array}{l}48.35 \\
(1.06)\end{array}$ & $\begin{array}{l}48 . \\
35 \\
(1.0 \\
6)\end{array}$ & $\begin{array}{l}0.00 \\
(0.00)\end{array}$ & $\begin{array}{l}51.73 \\
(0.73)\end{array}$ & $\begin{array}{l}0.00 \\
(0.00)\end{array}$ & $\begin{array}{l}51 . \\
73 \\
(0.7 \\
3)\end{array}$ \\
\hline $\begin{array}{l}\text { Sugarcane } \\
\text { based }\end{array}$ & $\begin{array}{l}0.0 \\
0 \\
(0.0 \\
0)\end{array}$ & $\begin{array}{l}0.00 \\
(0.00)\end{array}$ & $\begin{array}{l}0.0 \\
0 \\
(0.0 \\
0)\end{array}$ & $\begin{array}{l}0.0 \\
0 \\
(0.0 \\
0)\end{array}$ & $\begin{array}{l}0.00 \\
(0.0 \\
0)\end{array}$ & $\begin{array}{l}0.00 \\
(0.00)\end{array}$ & $\begin{array}{l}0.00 \\
(0.0 \\
0)\end{array}$ & $\begin{array}{l}0.00 \\
(0.0 \\
0)\end{array}$ & $\begin{array}{l}0.00 \\
(0.00 \\
)\end{array}$ & $\begin{array}{l}0.00 \\
(0.00)\end{array}$ & $\begin{array}{l}0.00 \\
(0.00 \\
)\end{array}$ & $\begin{array}{l}0.00 \\
(0.00 \\
)\end{array}$ & $\begin{array}{l}0.00 \\
(0.00)\end{array}$ & $\begin{array}{l}0.00 \\
(0.0 \\
0)\end{array}$ & $\begin{array}{l}0.00 \\
(0.00)\end{array}$ & $\begin{array}{l}0.00 \\
(0.0 \\
0)\end{array}$ & $\begin{array}{l}72.60 \\
(0.39)\end{array}$ & $\begin{array}{l}75 . \\
73 \\
(0.8 \\
0)\end{array}$ & $\begin{array}{l}78.75 \\
(1.39)\end{array}$ & $\begin{array}{l}77 . \\
31 \\
(0.8 \\
6)\end{array}$ & $\begin{array}{l}0.00 \\
(0.00)\end{array}$ & $\begin{array}{l}0.00 \\
(0.00)\end{array}$ & $\begin{array}{l}0.00 \\
(0.00)\end{array}$ & $\begin{array}{l}0.0 \\
0 \\
(0.0 \\
0)\end{array}$ \\
\hline Fibre based & $\begin{array}{l}0.0 \\
0 \\
(0.0 \\
0)\end{array}$ & $\begin{array}{l}0.00 \\
(0.00)\end{array}$ & $\begin{array}{l}0.0 \\
0 \\
(0.0 \\
0)\end{array}$ & $\begin{array}{l}0.0 \\
0 \\
(0.0 \\
0)\end{array}$ & $\begin{array}{l}43.4 \\
6 \\
(0.3 \\
77)\end{array}$ & $\begin{array}{l}39.80 \\
(0.97 \\
3)\end{array}$ & $\begin{array}{l}56.2 \\
3 \\
(2.6 \\
5)\end{array}$ & $\begin{array}{l}48.8 \\
6 \\
(1.3 \\
83)\end{array}$ & $\begin{array}{l}45.7 \\
4 \\
(0.61 \\
6)\end{array}$ & $\begin{array}{l}83.27 \\
(0.98 \\
5)\end{array}$ & $\begin{array}{l}29.6 \\
0 \\
(2.08 \\
2)\end{array}$ & $\begin{array}{l}45.0 \\
4 \\
(1.22 \\
8)\end{array}$ & $\begin{array}{l}46.29 \\
(0.396 \\
)\end{array}$ & $\begin{array}{l}0.00 \\
(0.0 \\
0)\end{array}$ & $\begin{array}{l}45.93 \\
(1.699)\end{array}$ & $\begin{array}{l}46.6 \\
0 \\
(1.2 \\
6)\end{array}$ & $\begin{array}{l}0.00 \\
(0.00)\end{array}$ & $\begin{array}{l}0.0 \\
0 \\
(0.0 \\
0)\end{array}$ & $\begin{array}{l}0.00 \\
(0.00)\end{array}$ & $\begin{array}{l}0.0 \\
0 \\
(0.0 \\
0)\end{array}$ & $\begin{array}{l}0.00 \\
(0.00)\end{array}$ & $\begin{array}{l}0.00 \\
(0.00)\end{array}$ & $\begin{array}{l}0.00 \\
(0.00)\end{array}$ & $\begin{array}{l}0.0 \\
0 \\
(0.0 \\
0)\end{array}$ \\
\hline $\begin{array}{l}\text { Dairy } \\
\text { based }\end{array}$ & $\begin{array}{l}48 . \\
82 \\
(0.4 \\
0)\end{array}$ & $\begin{array}{l}67.51 \\
(1.10)\end{array}$ & $\begin{array}{l}0.0 \\
0 \\
(0.0 \\
0)\end{array}$ & $\begin{array}{l}56 . \\
65 \\
(0.5 \\
2)\end{array}$ & $\begin{array}{l}0.00 \\
(0.0 \\
0)\end{array}$ & $\begin{array}{l}0.00 \\
(0.00)\end{array}$ & $\begin{array}{l}50.3 \\
9 \\
(1.6 \\
0)\end{array}$ & $\begin{array}{l}50.3 \\
9 \\
(1.6 \\
0)\end{array}$ & $\begin{array}{l}43.6 \\
2 \\
(0.59 \\
5)\end{array}$ & $\begin{array}{l}39.46 \\
(1.21 \\
3)\end{array}$ & $\begin{array}{l}39.3 \\
5 \\
(2.03 \\
1)\end{array}$ & $\begin{array}{l}41.1 \\
6 \\
(1.28 \\
0)\end{array}$ & $\begin{array}{l}0.00 \\
(0.00)\end{array}$ & $\begin{array}{l}64.0 \\
8 \\
(0.7 \\
92)\end{array}$ & $\begin{array}{l}61.16 \\
(1.47)\end{array}$ & $\begin{array}{l}62.4 \\
4 \\
(1.1 \\
31)\end{array}$ & $\begin{array}{l}0.00 \\
(0.40)\end{array}$ & $\begin{array}{l}49 . \\
82 \\
(0.0 \\
0)\end{array}$ & $\begin{array}{l}73.78 \\
(0.00)\end{array}$ & $\begin{array}{l}71 . \\
27 \\
(0.4 \\
0)\end{array}$ & $\begin{array}{l}0.00 \\
(0.00)\end{array}$ & $\begin{array}{l}0.00 \\
(0.00)\end{array}$ & $\begin{array}{l}0.00 \\
(0.00)\end{array}$ & $\begin{array}{l}0.0 \\
0 \\
(0.0 \\
0)\end{array}$ \\
\hline Fruit based & $\begin{array}{l}48 . \\
42 \\
(0.6 \\
0)\end{array}$ & $\begin{array}{l}42.88 \\
(1.40)\end{array}$ & $\begin{array}{l}59 . \\
56 \\
(2.4 \\
0)\end{array}$ & $\begin{array}{l}49 . \\
61 \\
(1.0 \\
9)\end{array}$ & $\begin{array}{l}65.0 \\
0 \\
(0.8)\end{array}$ & $\begin{array}{l}65.03 \\
(1.03 \\
3)\end{array}$ & $\begin{array}{l}47.7 \\
0 \\
(1.4 \\
7)\end{array}$ & $\begin{array}{l}57.4 \\
8 \\
(1.1 \\
6)\end{array}$ & $\begin{array}{l}0.00 \\
(0.00 \\
)\end{array}$ & $\begin{array}{l}0.00 \\
(0.00)\end{array}$ & $\begin{array}{l}0.00 \\
(0.00 \\
)\end{array}$ & $\begin{array}{l}0.00 \\
(0.00 \\
)\end{array}$ & $\begin{array}{l}0.00 \\
(0.00)\end{array}$ & $\begin{array}{l}54.4 \\
8 \\
(0.6 \\
16)\end{array}$ & $\begin{array}{l}0.00 \\
(0.00)\end{array}$ & $\begin{array}{l}54.4 \\
8 \\
(0.6 \\
16)\end{array}$ & $\begin{array}{l}0.00 \\
(0.00)\end{array}$ & $\begin{array}{l}0.0 \\
0 \\
(0.0 \\
0)\end{array}$ & $\begin{array}{l}0.00 \\
(0.00)\end{array}$ & $\begin{array}{l}0.0 \\
0 \\
(0.0 \\
0)\end{array}$ & $\begin{array}{l}50.04 \\
(0.45)\end{array}$ & $\begin{array}{l}0.00 \\
(0.00)\end{array}$ & $\begin{array}{l}0.00 \\
(0.00)\end{array}$ & $\begin{array}{l}50 . \\
04 \\
(0.4 \\
5)\end{array}$ \\
\hline $\begin{array}{l}\text { Piggery } \\
\text { based }\end{array}$ & $\begin{array}{l}53 . \\
21 \\
(0.3 \\
9)\end{array}$ & $\begin{array}{l}67.15 \\
(1.40)\end{array}$ & $\begin{array}{l}0.0 \\
0 \\
(0.0 \\
0)\end{array}$ & $\begin{array}{l}63 . \\
30 \\
(1.0 \\
2)\end{array}$ & $\begin{array}{l}0.00 \\
(0.0 \\
0)\end{array}$ & $\begin{array}{l}0.00 \\
(0.00)\end{array}$ & $\begin{array}{l}0.00 \\
(0.0 \\
0)\end{array}$ & $\begin{array}{l}0.00 \\
(0.0 \\
0)\end{array}$ & $\begin{array}{l}0.00 \\
(0.00 \\
)\end{array}$ & $\begin{array}{l}0.00 \\
(0.00)\end{array}$ & $\begin{array}{l}0.00 \\
(0.00 \\
)^{2}\end{array}$ & $\begin{array}{l}0.00 \\
(0.00 \\
)\end{array}$ & $\begin{array}{l}0.00 \\
(0.00)\end{array}$ & $\begin{array}{l}0.00 \\
(0.0 \\
0)\end{array}$ & $\begin{array}{l}0.00 \\
(0.00)\end{array}$ & $\begin{array}{l}0.00 \\
(0.0 \\
0)\end{array}$ & $\begin{array}{l}0.00 \\
(0.00)\end{array}$ & $\begin{array}{l}0.0 \\
0 \\
(0.0 \\
0)\end{array}$ & $\begin{array}{l}0.00 \\
(0.00)\end{array}$ & $\begin{array}{l}0.0 \\
0 \\
(0.0 \\
0)\end{array}$ & $\begin{array}{l}0.00 \\
(0.00)\end{array}$ & $\begin{array}{l}0.00 \\
(0.00)\end{array}$ & $\begin{array}{l}0.00 \\
(0.00)\end{array}$ & $\begin{array}{l}0.0 \\
0 \\
(0.0 \\
0)\end{array}$ \\
\hline $\begin{array}{l}\text { Fishery } \\
\text { based }\end{array}$ & $\begin{array}{l}0.0 \\
0 \\
(0.0 \\
0)\end{array}$ & $\begin{array}{l}0.00 \\
(0.00)\end{array}$ & $\begin{array}{l}0.0 \\
0 \\
(0.0 \\
0)\end{array}$ & $\begin{array}{l}0.0 \\
0 \\
(0.0 \\
0)\end{array}$ & $\begin{array}{l}0.00 \\
(0.0 \\
0)\end{array}$ & $\begin{array}{l}0.00 \\
(0.00)\end{array}$ & $\begin{array}{l}0.00 \\
(0.0 \\
0)\end{array}$ & $\begin{array}{l}0.00 \\
(0.0 \\
0)\end{array}$ & $\begin{array}{l}0.00 \\
(0.00 \\
)\end{array}$ & $\begin{array}{l}0.00 \\
(0.00)\end{array}$ & $\begin{array}{l}35.0 \\
5 \\
(2.14 \\
4)\end{array}$ & $\begin{array}{l}35.0 \\
5 \\
(0.71 \\
5)\end{array}$ & $\begin{array}{l}0.00 \\
(0.00)\end{array}$ & $\begin{array}{l}0.00 \\
(0.0 \\
0)\end{array}$ & $\begin{array}{l}0.00 \\
(0.00)\end{array}$ & $\begin{array}{l}0.00 \\
(0.0 \\
0)\end{array}$ & $\begin{array}{l}0.00 \\
(0.00)\end{array}$ & $\begin{array}{l}0.0 \\
0 \\
(0.0 \\
0)\end{array}$ & $\begin{array}{l}0.00 \\
(0.00)\end{array}$ & $\begin{array}{l}0.0 \\
0 \\
(0.0 \\
0)\end{array}$ & $\begin{array}{l}76.96 \\
(0.329)\end{array}$ & $\begin{array}{l}70.13 \\
(0.68 \\
1)\end{array}$ & $\begin{array}{l}57.45 \\
(1.483)\end{array}$ & $\begin{array}{l}68 . \\
18 \\
(0.6 \\
1)\end{array}$ \\
\hline $\begin{array}{l}\text { Plantation } \\
\text { based }\end{array}$ & $\begin{array}{l}47 . \\
85 \\
(0.3 \\
8)\end{array}$ & $\begin{array}{l}58.75 \\
(1.47)\end{array}$ & $\begin{array}{l}0.0 \\
0 \\
(0.0 \\
0)\end{array}$ & $\begin{array}{l}55 . \\
50 \\
(0.8 \\
4)\end{array}$ & $\begin{array}{l}0.00 \\
(0.0 \\
0)\end{array}$ & $\begin{array}{l}0.00 \\
(0.00)\end{array}$ & $\begin{array}{l}0.00 \\
(0.0 \\
0)\end{array}$ & $\begin{array}{l}0.00 \\
(0.0 \\
0)\end{array}$ & $\begin{array}{l}83.8 \\
2 \\
(0.75 \\
5)\end{array}$ & $\begin{array}{l}86.97 \\
(1.56 \\
8)\end{array}$ & $\begin{array}{l}81.4 \\
8 \\
(2.25 \\
3)\end{array}$ & $\begin{array}{l}83.6 \\
2 \\
(1.52 \\
5)\end{array}$ & $\begin{array}{l}0.00 \\
(0.00)\end{array}$ & $\begin{array}{l}0.00 \\
(0.0 \\
0)\end{array}$ & $\begin{array}{l}0.00 \\
(0.00)\end{array}$ & $\begin{array}{l}0.00 \\
(0.0 \\
0)\end{array}$ & $\begin{array}{l}0.00 \\
(0.00)\end{array}$ & $\begin{array}{l}0.0 \\
0 \\
(0.0 \\
0)\end{array}$ & $\begin{array}{l}0.00 \\
(0.00)\end{array}$ & $\begin{array}{l}0.0 \\
0 \\
(0.0 \\
0)\end{array}$ & $\begin{array}{l}83.50 \\
(0.44)\end{array}$ & $\begin{array}{l}85.14 \\
(0.78)\end{array}$ & $\begin{array}{l}79.78 \\
(1.01)\end{array}$ & $\begin{array}{l}83 . \\
41 \\
(0.6 \\
5)\end{array}$ \\
\hline $\begin{array}{l}\text { Spices } \\
\text { based }\end{array}$ & $\begin{array}{l}34 . \\
94 \\
(0.8 \\
0)\end{array}$ & $\begin{array}{l}0.00 \\
(0.00)\end{array}$ & $\begin{array}{l}0.0 \\
0 \\
(0.0 \\
0)\end{array}$ & $\begin{array}{l}34 . \\
94 \\
(0.8 \\
0)\end{array}$ & $\begin{array}{l}0.00 \\
(0.0 \\
0)\end{array}$ & $\begin{array}{l}0.00 \\
(0.00)\end{array}$ & $\begin{array}{l}0.00 \\
(0.0 \\
0)\end{array}$ & $\begin{array}{l}0.00 \\
(0.0 \\
0)\end{array}$ & $\begin{array}{l}0.00 \\
(0.00 \\
)\end{array}$ & $\begin{array}{l}0.00 \\
(0.00)\end{array}$ & $\begin{array}{l}0.00 \\
(0.00 \\
)\end{array}$ & $\begin{array}{l}0.00 \\
(0.00 \\
)\end{array}$ & $\begin{array}{l}0.00 \\
(0.00)\end{array}$ & $\begin{array}{l}0.00 \\
(0.0 \\
0)\end{array}$ & $\begin{array}{l}0.00 \\
(0.00)\end{array}$ & $\begin{array}{l}0.00 \\
(0.0 \\
0)\end{array}$ & $\begin{array}{l}0.00 \\
(0.00)\end{array}$ & $\begin{array}{l}0.0 \\
0 \\
(0.0 \\
0)\end{array}$ & $\begin{array}{l}0.00 \\
(0.00)\end{array}$ & $\begin{array}{l}0.0 \\
0 \\
(0.0 \\
0)\end{array}$ & $\begin{array}{l}0.00 \\
(0.00)\end{array}$ & $\begin{array}{l}0.00 \\
(0.00)\end{array}$ & $\begin{array}{l}0.00 \\
(0.00)\end{array}$ & $\begin{array}{l}0.0 \\
0 \\
(0.0 \\
0)\end{array}$ \\
\hline $\begin{array}{l}\text { Others } \\
\text { based }\end{array}$ & $\begin{array}{l}0.0 \\
0 \\
(0.0 \\
0)\end{array}$ & $\begin{array}{l}0.00 \\
(0.00)\end{array}$ & $\begin{array}{l}0.0 \\
0 \\
(0.0 \\
0)\end{array}$ & $\begin{array}{l}0.0 \\
0 \\
(0.0 \\
0)\end{array}$ & $\begin{array}{l}50.1 \\
4 \\
(0.5 \\
43)\end{array}$ & $\begin{array}{l}43.83 \\
(1.2)\end{array}$ & $\begin{array}{l}76.1 \\
0 \\
(1.6)\end{array}$ & $\begin{array}{l}54.5 \\
8 \\
(0.7 \\
33)\end{array}$ & $\begin{array}{l}54.0 \\
4 \\
(0.69 \\
8)\end{array}$ & $\begin{array}{l}39.54 \\
(1.64 \\
4)\end{array}$ & $\begin{array}{l}0.00 \\
(0.00 \\
)\end{array}$ & $\begin{array}{l}45.5 \\
7 \\
(0.78 \\
0)\end{array}$ & $\begin{array}{l}0.00 \\
(0.00)\end{array}$ & $\begin{array}{l}0.00 \\
(0.0 \\
0)\end{array}$ & $\begin{array}{l}0.00 \\
(0.00)\end{array}$ & $\begin{array}{l}0.00 \\
(0.0 \\
0)\end{array}$ & $\begin{array}{l}0.00 \\
(0.00)\end{array}$ & $\begin{array}{l}0.0 \\
0 \\
(0.0 \\
0)\end{array}$ & $\begin{array}{l}0.00 \\
(0.00)\end{array}$ & $\begin{array}{l}0.0 \\
0 \\
(0.0 \\
0)\end{array}$ & $\begin{array}{l}65.36 \\
(0.34)\end{array}$ & $\begin{array}{l}71.26 \\
(0.78)\end{array}$ & $\begin{array}{l}57.27 \\
(1.36)\end{array}$ & $\begin{array}{l}66 . \\
12 \\
(0.6 \\
3)\end{array}$ \\
\hline
\end{tabular}

Figures in the parentheses indicates respective average land holding size (ha.) of different size classes

\subsection{Economic Analysis of Profitable Sub Farming Systems}

Economic analysis of different sub-systems exhibited higher economic return from Dairy (Econ. B: C 6.93), Plantation (Econ. B: C 6.75) and Piggery-based farming systems (Econ. B: C 6.06) than existing cropping systems in Hilly tracts of Bengal. Vegetable became an income generating systems in almost all the regions of the state and vegetable based sub-systems registered higher economic return in Lateritic red soil zone (Econ. B: C 4.76), Coastal saline belt (Econ. B: C 7.02), Old alluvial zone (Econ. B: C 6.29), New alluvium or Gangetic alluvium tract (Econ. B: C 3.69) and Terai zone (Econ. B: C 4.44). Orange became a profitable fruit crop in Hilly tracts, where orange based sub-farming systems exhibited economic return of 3.46. Sugarcane became popular in Lateritic red soil region that generated a return of 2.85. Fishery and betel vine-based sub-farming systems dominated in the Coastal saline zone of the state, exhibiting an economic return of 4.42 and 3.88 respectively. Baruipur sub-division under Coastal saline zone is famous for guava cultivation where fruit-based farming systems showed high economic return (6.44) in this region. Jute cultivators dominated the north Bengal and Gangetic alluvium tract of the state, but Terai zone exhibited better economic return from this short-duration cash crop (Econ. B: C 3.33). Old alluvium tract exhibited an Economic B:C of 2.60 for jute-based sub-systems. New alluvial zone is famous for vegetables cultivation and orchard (Banana and Papaya). Fruit based sub-systems also exhibited a higher economic return (3.99) (Table 3). All types of farmers in several pockets of the state have shifted to vegetables and fruit cultivation, owing to better irrigation and extension support, in recent times (Mitra and Pathak, 2008). Overall, diversified farms showed a relatively higher cost-benefit ratio. Increasing dependence on costly external inputs has rendered an increase in cost of cultivation for less diversified farms, relative to system net return, irrespective of farm types [13]. 
Table 3: Farm size-wise economically most profitable different sub-farming systems in all NARP zones of West Bengal

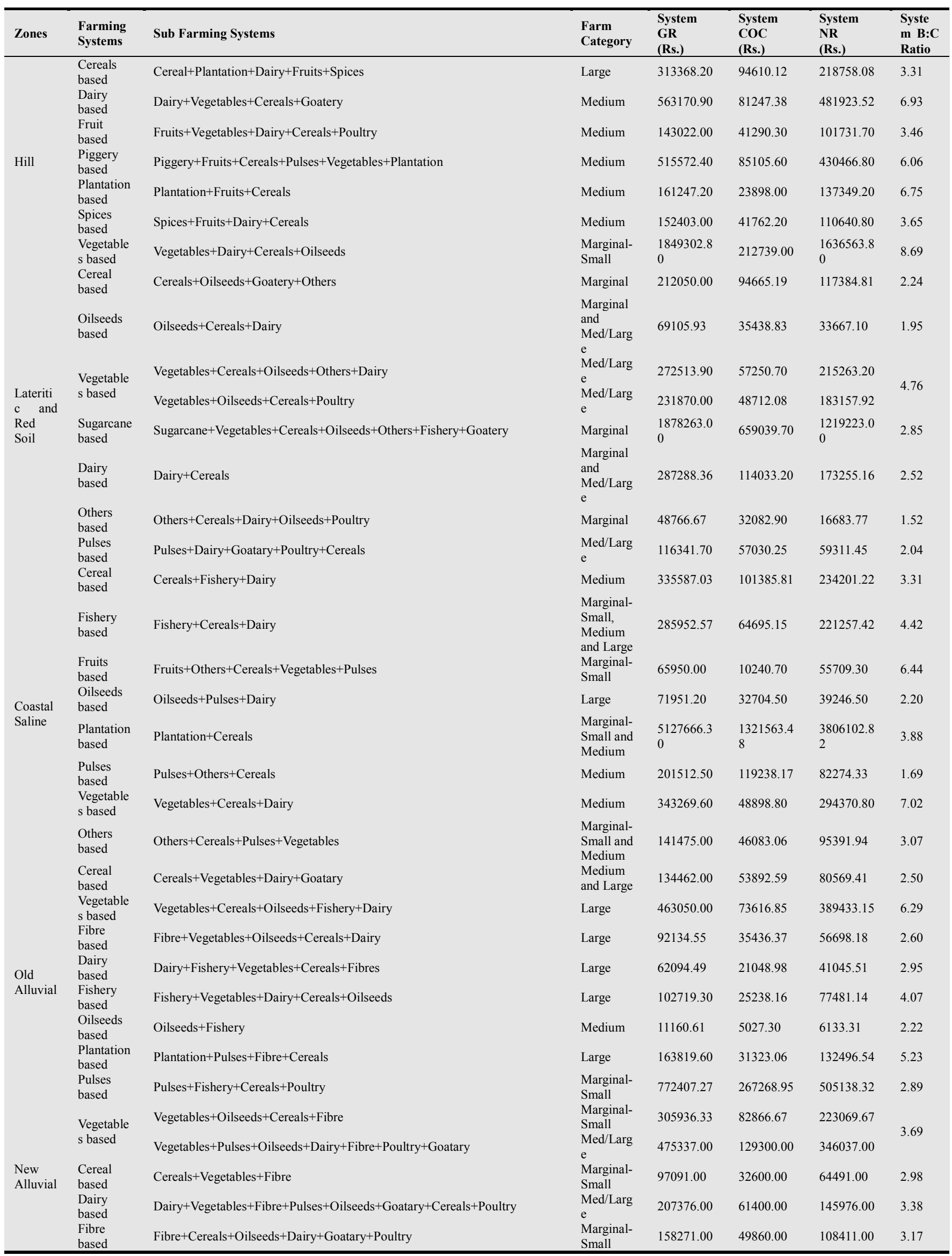




\begin{tabular}{|c|c|c|c|c|c|c|c|}
\hline Zones & $\begin{array}{l}\text { Farming } \\
\text { Systems }\end{array}$ & Sub Farming Systems & $\begin{array}{l}\text { Farm } \\
\text { Category }\end{array}$ & $\begin{array}{l}\text { System } \\
\text { GR } \\
\text { (Rs.) } \\
\end{array}$ & $\begin{array}{l}\text { System } \\
\text { COC } \\
\text { (Rs.) }\end{array}$ & $\begin{array}{l}\text { System } \\
\text { NR } \\
\text { (Rs.) }\end{array}$ & $\begin{array}{l}\text { Syste } \\
\text { m B:C } \\
\text { Ratio }\end{array}$ \\
\hline \multirow{7}{*}{ Terai } & $\begin{array}{l}\text { Fruits } \\
\text { based }\end{array}$ & Fruits + Vegetables + Dairy + Oilseeds + Cereals + Fibre + Goatary + Poultry & $\begin{array}{l}\text { Marginal- } \\
\text { Small }\end{array}$ & 815521.00 & 204530.00 & 610991.00 & 3.99 \\
\hline & $\begin{array}{l}\text { Cereal } \\
\text { based }\end{array}$ & Cereals + Pulses + Fibre + Fruits + Vegetables + Poultry + Piggery & Large & 163080.00 & 56765.00 & 106315.00 & 2.87 \\
\hline & $\begin{array}{l}\text { Vegetable } \\
\text { s based }\end{array}$ & Vegetables + Cereals + Fibre + Fruits + Livestock + Oilseeds + Spices + Goatary & Large & 257700.00 & 58044.00 & 199656.00 & 4.44 \\
\hline & $\begin{array}{l}\text { Fibre } \\
\text { based }\end{array}$ & Fibre + Cereals + Others + Vegetables + Livestock + Fruits & Medium & 131300.00 & 39400.00 & 91900.00 & 3.33 \\
\hline & $\begin{array}{l}\text { Fruits } \\
\text { based }\end{array}$ & Fruits + Cereals + Livestock + Poultry & Medium & 98200.00 & 22333.00 & 75867.00 & 4.40 \\
\hline & $\begin{array}{l}\text { Livestock } \\
\text { based }\end{array}$ & Livestock + Vegetables + Cereals + Fibre + Fruits + Oilseeds & Large & 918476.00 & 256554.00 & 661922.00 & 3.58 \\
\hline & $\begin{array}{l}\text { Others } \\
\text { based }\end{array}$ & $\begin{array}{l}\text { Others }+ \text { Livestock }+ \text { Cereals }+ \text { Fibre }+ \text { Fruits }+ \text { Vegetables }+ \text { Oilseeds }+ \text { Poultry }+ \text { Puls } \\
\text { es }\end{array}$ & Medium & 252195.00 & 63782.00 & 188413.00 & 3.95 \\
\hline
\end{tabular}

Note: GR: Gross Return, COC: Cost of Cultivation, NR: Net Return, B:C ratio: Benefit Cost ratio

\subsection{Extent of Crop Diversification among all Size Classes}

The paper also evaluates and assesses the extent of crop diversification among different size classes using Herfindahal index across all agro-climatic zones of West Bengal (Table 4). Marginal-small farming community diversified their cropping pattern to a more income generating system such as short term vegetables, fruit orchard, fishery, dairy and piggery as compared to large farmers. In Hilly tracts, extent of diversification among marginal and small farming community were 0.330 and 0.341 respectively. This was due to the cultivation of winter and summer vegetables, orange as fruit, development of cattle-based enterprise and piggery as alternative sources of income. The extent of diversification varied from 0.161 to 0.500 for marginal farmers and 0.155 to 0.500 for the medium farmers. However, the extent of crop diversification among marginal and small farm families reached its maximum in Terai region (1.000), Old alluvium tract of the river Ganges and dry tract (Lateritic red soil zone) of West Bengal indicating significant shift in cropping pattern. This shift was from low-value/subsistence to high-value crop, supported by technology adoption and extension support. The picture is more or less similar in rest of the zones. Medium size category of farmers in New alluvial zone diversified their cropping system $(0.505)$ to vegetables-based sub systems for more income generation. Small (0.646) as well as large (0.649) farming community diversified their cropping in Coastal saline belt of Bengal towards more income generating fishery and plantation (betel vine) based systems to raise their overall livelihood status. Interestingly, smaller farms have shown higher diversification in income which also is in line with available literature $[14,15]$.

Table 4: Extent of Crop Diversification among different size classes across all agro-climatic zones of West Bengal

\begin{tabular}{|c|c|c|c|c|c|c|c|c|c|c|c|c|c|c|c|c|c|c|}
\hline \multirow[t]{3}{*}{$\begin{array}{l}\text { Categ } \\
\text { ory }\end{array}$} & \multicolumn{3}{|c|}{ Hill Zone } & \multicolumn{3}{|c|}{ Terai Zone } & \multicolumn{3}{|c|}{ Old Alluvial Zone } & \multicolumn{3}{|c|}{ New Alluvial Zone } & \multicolumn{2}{|c|}{$\begin{array}{l}\text { Lateritic } \\
\text { Soil Zone }\end{array}$} & \multirow[t]{2}{*}{ Red } & \multicolumn{2}{|c|}{$\begin{array}{l}\text { Coastal } \\
\text { Zone }\end{array}$} & \multirow{3}{*}{$\begin{array}{l}\text { Saline } \\
\text { Avera } \\
\text { ge }\end{array}$} \\
\hline & Min & $\mathrm{Ma}$ & & Min & $\mathrm{Ma}$ & & Min & $\mathrm{Ma}$ & & Min & $\mathrm{Ma}$ & & Min & $\mathrm{Ma}$ & & Min & $\mathrm{Ma}$ & \\
\hline & $\begin{array}{l}\text { Val } \\
\text { ue }\end{array}$ & $\begin{array}{l}\text { x. } \\
\text { Val } \\
\text { ue }\end{array}$ & $\begin{array}{l}\text { Avera } \\
\text { ge }\end{array}$ & $\begin{array}{l}\text { Val } \\
\text { ue }\end{array}$ & $\begin{array}{l}\text { x. } \\
\text { Val } \\
\text { ue }\end{array}$ & $\begin{array}{l}\text { Avera } \\
\text { ge }\end{array}$ & $\begin{array}{l}\text { Val } \\
\text { ue }\end{array}$ & $\begin{array}{l}\text { x. } \\
\text { Val } \\
\text { ue }\end{array}$ & $\begin{array}{l}\text { Avera } \\
\text { ge }\end{array}$ & $\begin{array}{l}\text { Val } \\
\text { ue }\end{array}$ & $\begin{array}{l}\text { x. } \\
\text { Val } \\
\text { ue }\end{array}$ & $\begin{array}{l}\text { Avera } \\
\text { ge }\end{array}$ & $\begin{array}{l}\text { Val } \\
\text { ue }\end{array}$ & $\begin{array}{l}\text { x. } \\
\text { Val } \\
\text { ue }\end{array}$ & $\begin{array}{l}\text { Avera } \\
\text { ge }\end{array}$ & $\begin{array}{l}\text { Val } \\
\text { ue }\end{array}$ & $\begin{array}{l}\text { x. } \\
\text { Val } \\
\text { ue }\end{array}$ & \\
\hline $\begin{array}{l}\text { Margi } \\
\text { nal }\end{array}$ & $\begin{array}{l}0.1 \\
61\end{array}$ & $\begin{array}{l}0.5 \\
00\end{array}$ & 0.330 & $\begin{array}{l}0.2 \\
96\end{array}$ & $\begin{array}{l}1.0 \\
00\end{array}$ & 0.648 & $\begin{array}{l}0.3 \\
32\end{array}$ & $\begin{array}{l}1.0 \\
00\end{array}$ & 0.666 & $\begin{array}{l}0.1 \\
90\end{array}$ & $\begin{array}{l}0.5 \\
00\end{array}$ & 0.345 & $\begin{array}{l}0.2 \\
34\end{array}$ & $\begin{array}{l}1.0 \\
00\end{array}$ & 0.617 & $\begin{array}{l}0.2 \\
50\end{array}$ & $\begin{array}{l}0.7 \\
22\end{array}$ & 0.486 \\
\hline Small & $\begin{array}{l}0.2 \\
53\end{array}$ & $\begin{array}{l}0.4 \\
30\end{array}$ & 0.341 & $\begin{array}{l}0.3 \\
49\end{array}$ & $\begin{array}{l}1.0 \\
00\end{array}$ & 0.675 & $\begin{array}{l}0.2 \\
13\end{array}$ & $\begin{array}{l}0.2 \\
14\end{array}$ & 0.213 & $\begin{array}{l}0.1 \\
50\end{array}$ & $\begin{array}{l}0.3 \\
00\end{array}$ & 0.225 & $\begin{array}{l}0.3 \\
21\end{array}$ & $\begin{array}{l}1.0 \\
00\end{array}$ & 0.660 & $\begin{array}{l}0.5 \\
10\end{array}$ & $\begin{array}{l}0.7 \\
83\end{array}$ & 0.646 \\
\hline $\begin{array}{l}\text { Mediu } \\
\mathrm{m}\end{array}$ & $\begin{array}{l}0.1 \\
55\end{array}$ & $\begin{array}{l}0.5 \\
00\end{array}$ & 0.327 & $\begin{array}{l}0.3 \\
05\end{array}$ & $\begin{array}{l}0.5 \\
45\end{array}$ & 0.425 & $\begin{array}{l}0.3 \\
28\end{array}$ & $\begin{array}{l}0.5 \\
51\end{array}$ & 0.439 & $\begin{array}{l}0.0 \\
70\end{array}$ & $\begin{array}{l}0.9 \\
40\end{array}$ & 0.505 & $\begin{array}{l}0.3 \\
21\end{array}$ & $\begin{array}{l}1.0 \\
00\end{array}$ & 0.660 & $\begin{array}{l}0.4 \\
01\end{array}$ & $\begin{array}{l}0.6 \\
37\end{array}$ & 0.519 \\
\hline Large & $\begin{array}{l}0.3 \\
14\end{array}$ & $\begin{array}{l}0.3 \\
34\end{array}$ & 0.324 & $\begin{array}{l}0.5 \\
86\end{array}$ & $\begin{array}{l}0.7 \\
82\end{array}$ & 0.684 & $\begin{array}{l}0.2 \\
70\end{array}$ & $\begin{array}{l}0.4 \\
99\end{array}$ & 0.384 & $\begin{array}{l}0.1 \\
60\end{array}$ & $\begin{array}{l}0.2 \\
40\end{array}$ & 0.200 & $\begin{array}{l}0.2 \\
33\end{array}$ & $\begin{array}{l}0.7 \\
30\end{array}$ & 0.481 & $\begin{array}{l}0.4 \\
35\end{array}$ & $\begin{array}{l}0.8 \\
63\end{array}$ & 0.649 \\
\hline
\end{tabular}

\section{Conclusions}

Across all agro-climatic zones of West Bengal, farming community of all size classes started to shift from their traditional rice-based cropping system to more income generating systems based on fruits, vegetables, livestock, plantation, spices and piggery. Piggery-based sub-systems in Hilly tract, fibre- and vegetables-based systems in Terai as well as Old alluvium regions, vegetables in New alluvial, sugarcane and vegetables in Lateritic red soil zone and fishery, oilseeds and plantation (betel vine) in the Coastal belt of the state. However, Herfindahal crop diversification measurement showed a tendency of marginal, small and medium farmers to diversify their cropping pattern. The range varied widely among the different size classes of farmers indicating complete diversification to perfect specialization to traditional farming system. This could have been possible only due to facilitation with better knowledge and proper intervention by agricultural extension. New alluvial zone, owing to its proximity to Bidhan Chandra Krishi Viswavidyalaya, the sole State Agricultural University in southern Bengal, demonstrated a 
stronger departure from rice-based farming than other zones, and that was most pronounce among marginal farmers. Small and marginal farmers were still preoccupied with their land with little off-farm shift in livelihoods. Medium and large farmers had already diversified into other occupations, and contrary to belief, farming might have become a secondary occupation for them. This saw less diversification among this class.

Among the crops, rice showed a diverse shift to several sub-farming systems. Jute was still predominant in traditional pockets and showed stability of acreage and production. Fruits and vegetables were on the rise across agro-ecological zones and size categories. Livestock, fishery, piggery and plantation were incorporated in specific farming systems of specific areas. This scenario is welcome. Farmers across all regions had perhaps become largely conscious that water and other inputs had a greater return when invested in fruits and vegetables compared to rice and other agronomic crops. Jute crop, despite its oddities with price, was more of agronomic compulsion. Moreover, jute rendered more benefits with vegetables than rice in the same piece of land.

Livestock was also on the rise along with fishery in the problematic coastal saline belt. This also is a welcome shift embodying diversification in enterprises. With gradual departure from rice and other cereals and shift favouring fruits and orchard enterprise, and livestock and fishery, better productivity and remuneration per unit of land is imminent and this might be providing enhanced opportunity of employment with less gestation.

\section{Acknowledgements}

For performing the entire survey work the contribution and assistance of all the sampled farming communities, block and district officials, Assistant Director of Agriculture of the respective blocks are being duly acknowledged. Further, the Project Directorate of Farming System Research, ICAR is being duly acknowledged for providing funds for the entire survey work across all the zones of West Bengal, India.

\section{References}

[1] FAO. 1990. Farming Systems Development: Guidelines for the conduct of a training course in Farming Systems Development. Rome, Italy.

[2] Dixon, J., Gulliver, A., Gibbon, D. 2001. Farming systems and poverty: improving farmers' livelihoods in a changing world. Rome: FAO.

[3] Chambers, R., Pacey, A., Thrupp, L.A. 1989. Farmer first: farmer innovation and agricultural research, London: Intermediate Technology Publications.
[4] Ojiem, J., Ridder, N., Vanlauwe, B., Giller, K.E. 2006. Socio-ecological niche: a conceptual framework for integration of legumes in smallholder farming systems. International Journal of Agric. Sustainability. 4(1), 79-93.

[5] Ganpat, W., Bekele, I. 2001. Looking for the trees in the forest: farm typology as a useful tool in defining targets for extension. In: Emerging Trends in Agricultural and Extension Education (J.R. Lindner, ed.), Proceedings of the 17th Annual Conference of the Association for International Agricultural and Extension Education. 4-7 Arpil 2001, Baton Rouge, Louisiana.

[6] Timothy, W.O. 1994. Identifying target groups for livestock improvement research: The classification of sedentary livestock producers in western Niger. Agricultural Systems. $46,227-237$.

[7] Vanclay, J.K. 2005. Using a typology of tree-growers to guide forestry extension. Annals of Tropical Research. 27(1), 97-103.

[8] Tittonell, P., Muriukid, A., Shepherde, K.D., Mugendif, D., Kaizzig, K.C., Okeyoa, J., Verchote, L., Coee, R., Vanlauwea, B. 2010. The diversity of rural livelihoods and their influence on soil fertility in agricultural systems of East Africa - A typology of smallholder farms. Agricultural Systems. 103 (2), 83-97.

[9] Goswami, R. 2007. Understanding farmer-to-farmer communication within the Sustainable Rural Livelihood framework. Thesis $\mathrm{PhD}$, Bidhan Chandra Krishi Viswavidyalaya, Department of Agricultural Extension, West Bengal, India.

[10] Mehta, R. 2009. Rural livelihood diversification and its measurement issues: focus India. Wye City Group on statistics on rural development and agriculture household income, Second Meeting, at FAO HQ, Italy, Rome, 11-12 June 2009. Available at www.fao.org/fileadmin/templates/ess/pages/rural/wye_city_ group/2009/

[11] De, U.K. 2000. Diversification of Crop in West Bengal: A Spatio-Temporal Analysis, Artha Vijnana, 42: 170-182.

[12] Bagchi, B. D., S. B. Roy, W. M. H. Jaim and Hossain, M. 2012. Diversity, spatial distribution, and the process of adoption of improved rice varieties in West Bengal, In: M. Hossain, W.M.H. Jaim, T.R. Paris and B. Hardy (Editors) Adoption and diffusion of modern rice varieties in Bangladesh and eastern India. IRRI, Philippines, pp 31-44.

[13] Vyas, V. S. 2001. Agriculture: second round of economic reforms, Economic and Political Weekly, 36: 829-836.

[14] De, U.K. 2003. Economics of crop diversification. Akansha Pub. House, New Delhi.

[15] Joshi, P.K., A. Gulati and R. Cummings Jr. 2007. Agricultural Diversification and Smallholders in South Asia. Academic Foundation, New Delhi, India. 